\title{
Simulation of Gas- and Liquid-Phase Layer-By-Layer Deposition of Metal Oxides by Coarse-Grained Modeling
}

\author{
Kristopher S. Brown, ${ }^{\dagger}$ Chiara Saggese, ${ }^{\ddagger}{ }^{\circ}$ Benjamin P. Le Monnier, Florent Héroguel, \\ and Jeremy S. Luterbacher*(1)
}

Laboratory of Sustainable and Catalytic Processing, Institute of Chemical Sciences and Engineering, École Polytechnique Fédérale de Lausanne (EPFL), CH-1015 Lausanne, Switzerland

\section{Supporting Information}

ABSTRACT: Atomic layer deposition (ALD) and chemical layer deposition (CLD) are techniques to produce conformal, atomically precise overcoats for materials in gas and liquid phases, respectively. Although it is a challenge to explicitly model ALD/CLD dynamics because of the large timescales involved, a combination of rulebased deposition actions, followed by molecular dynamics relaxations, can capture the net effect of one cycle. For ALD, we developed deposition rules for the trimethylaluminum (TMA) $/ \mathrm{H}_{2} \mathrm{O}$ system over $\mathrm{Al}_{2} \mathrm{O}_{3}$ and $\mathrm{SiO}_{2}$ substrates. We also explore the CLD system of $\mathrm{Al}(\mathrm{O} \text {-sec- } \mathrm{Bu})_{3} / \mathrm{H}_{2} \mathrm{O}$ solvated in 2-butanol over an $\mathrm{Al}_{2} \mathrm{O}_{3}$ substrate. In contrast to previous studies focusing on ligand exchange reactions, the gas-phase simulations predict that a TMA

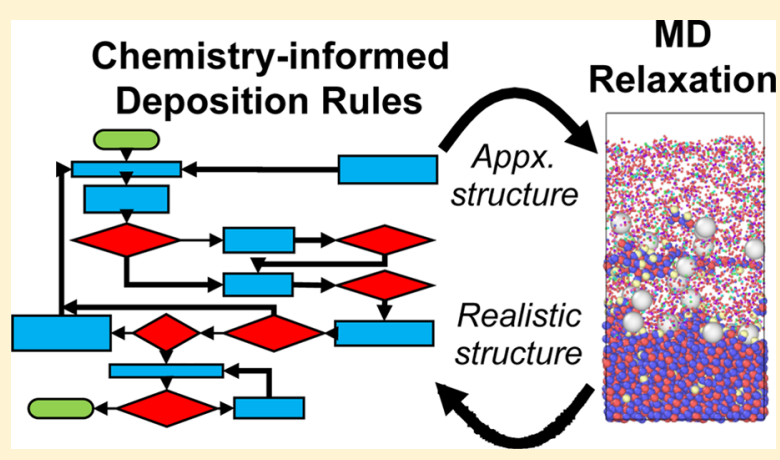
dissociation mechanism is the dominant growth mode, whereas the CLD simulations show a primary growth mode of deposition through precipitation of species that have already undergone reactions in solution. Together, these modeling results constitute a refinement of preexisting ALD models, leading to more accurate growth rate predictions, as well as a first step toward modeling more challenging liquid-phase processes.

\section{INTRODUCTION}

Atomic layer deposition (ALD) is a technique for depositing thin films, capable of producing uniform and conformal overcoats with atomically precise control over the film thickness and composition. ${ }^{1-3}$ The film growth proceeds through alternating self-terminating heterogeneous reactions above a solid substrate. These characteristics make ALD useful for several applications in the fields of microelectronics, catalysis, and energy conversion, storage, and utilization. ${ }^{2,4-6}$ Catalyst overcoating by deposition of a metal oxide layer is a promising method to curtail irreversible deactivation. Several overcoating methods have been shown to protect metal nanoparticles against sintering and leaching. These methods include the use of ALD of alumina ${ }^{7,8}$ and silica deposition by sol-gel. ${ }^{9,10}$

ALD processes consist of sequential alternating gas-phase pulses of chemical precursors that react with the substrate. Each sequential gas-surface reactions is referred to as a "half cycle", during which excess precursor is pulsed into a chamber under reduced pressure for enough time to allow saturation of the substrate surface through a self-limiting process. Subsequently, unreacted precursor or reaction byproducts are eliminated by purging with an inert carrier. Because of the self-limiting nature of the reaction, the two half cycles leave no more than one monolayer of the material at the surface. This process is then cycled until the appropriate film thickness is achieved. ${ }^{11}$
Among several ALD processes, the deposition of aluminum oxide, $\mathrm{Al}_{2} \mathrm{O}_{3}$, using trimethylaluminum (TMA) and water as precursors, is the most studied ALD process to date and is considered as a model process for ALD. ${ }^{1}$ This is mainly due to the ideally self-terminating nature of the adsorption process and the inertness of methane-the reaction's by-product. Moreover, ALD's production of aluminum oxide, which is a high- $k$ dielectric material, is also of great interest for microelectronics. $^{12}$

Common gas-phase deposition techniques have limitations which can be addressed by carrying out ALD chemistry in the liquid phase. These limitations include the restricted types of precursors and their byproducts that must be gaseous at feasible temperatures and the requirement of near-vacuum conditions. ${ }^{12}$ Furthermore, in the case of coating 3D substrates, a specialized equipment is required, such as fluidized bed reactors to ensure diffusion of precursors into substrate pores. Liquid-phase synthesis techniques can mimic the gas-phase process at near room temperatures using a simple wet chemistry equipment by sequentially exposing a substrate to stoichiometric amounts of the metal and liquid precursors or by using an excess of precursors with the washing/drying step in between each cycle. $^{13-17}$

Received: January 7, 2018

Published: February 26, 2018 
1.1. ALD Gas-Phase Technique. $\mathrm{Al}_{2} \mathrm{O}_{3} \mathrm{ALD}$ growth using TMA and $\mathrm{H}_{2} \mathrm{O}$ can be represented by the following two half reactions (eqs 1 and 2)

$$
\begin{aligned}
& \mathrm{AlOH}+\mathrm{Al}\left(\mathrm{CH}_{3}\right)_{3} \rightarrow \mathrm{Al}-\mathrm{O}-\mathrm{Al}\left(\mathrm{CH}_{3}\right)_{2}+\mathrm{CH}_{4} \\
& \mathrm{Al}-\mathrm{O}-\mathrm{Al}\left(\mathrm{CH}_{3}\right)_{2}+2 \mathrm{H}_{2} \mathrm{O} \rightarrow \mathrm{Al}-\mathrm{O}-\mathrm{Al}(\mathrm{OH})_{2}+2 \mathrm{CH}_{4}
\end{aligned}
$$

The network of elementary reactions is far more complex, and numerous studies have examined the reaction details. $1,18-20$ Despite the widespread use of ALD, the details of its physical and chemical processes are not yet well-understood and continue to be the focus of both experimental and numerical studies. ${ }^{21}$ Experimentally researchers improve the deposited films by adjusting operating conditions, such as process pressure, temperature, and dosage. ${ }^{22-25}$ Recently, advances in computing technologies have allowed studying physical interactions and chemical kinetics of ALD by numerical approaches.

ALD is intrinsically a multiscale process because it is manifest across many length and time scales, ${ }^{21,26}$ and the primary challenge to model ALD is to effectively combine different scales according to the property of interest. Indeed, the pulsed flow of gases into meter-scale reactors, around millimeter-scaled geometries, leads to chemical reactions between atoms, which aggregate into nanometer-thick films and coat micrometerscaled pores or particles. The problem of timescale in ALD is perhaps even more acute than that of length scale because a combination of fast (picoseconds-nanoseconds) and slow reactions (microseconds-milliseconds) contributes to the film growth, and the pressure changes as gases are pulsed and purged over second-long timescales.

On the molecular scale, most numerical investigations adopt first-principles methods, such as density functional theory (DFT), to explore surface reaction mechanisms and chemical reaction pathways. ${ }^{20,27}$ These atomic-level models reveal certain microscopic details of the surface reaction mechanisms and chemical kinetics but fail to account for the multiscale nature of ALD and are not directly applicable to engineering applications. Other researchers take the macroscopic transport processes into consideration, using Monte Carlo methods, lattice Boltzmann methods, and molecular dynamics (MD). Kinetic Monte Carlo (KMC) is an approach that combines reaction events with different timescales to reveal the evolution of the overall process in time. The lattice KMC (LKMC) method was successfully applied to the modeling of $\mathrm{Hf}\left(\mathrm{N}\left(\mathrm{CH}_{3}\right)_{2}\right)_{4} / \mathrm{H}_{2} \mathrm{O}$ $\mathrm{ALD}^{28}$ The LKMC method requires the complete knowledge of reaction pathways, as well as their rate constants, which can be calculated ab initio from transition-state theory and DFT. The KMC algorithm uses this information to simulate the system evolution by randomly executing events at all timescales, with event probabilities proportional to their rate constants. $\mathrm{Hu}$ et al. ${ }^{29}$ presented an alternative strategy that employs classical MD. MD alone cannot model entire ALD cycles because of the limited simulation timescales for large systems, so $\mathrm{Hu}$ et al. bypassed this timescale limitation by coupling MD with a set of deposition rules.

Deposition rules can be described as a function that accepts an uncoated surface as input and returns an approximated coated surface as output. MD relaxation steps correct these approximations to produce a realistic surface structure, which serves as the input into the deposition rules of the next cycle. An inherent advantage of the MD method is found in its lack of lattice constraints, allowing it to better represent amorphous structures. Overall, the combination of $\mathrm{MD}$ with rule-based deposition makes a favorable compromise between the computationally intensive explicit modeling of the deposition process with the overly simplified modeling of ALD at a macroscopic scale. We extend the previous work by $\mathrm{Hu}$ et al. ${ }^{29}$ in this direction by adding more complexity to the rule-based process, allowing us to explore most of the possible chemical mechanisms and modes of growth.

1.2. Liquid-Phase Technique. Motivated by the challenge to develop a practical synthesis technique for creating catalysts resistant to sintering, poisoning, and leaching, we recently developed a deposition process involving $\mathrm{Al}(\mathrm{O}-\mathrm{sec}-\mathrm{Bu}) / \mathrm{H}_{2} \mathrm{O}$ solvated in 2-butanol, which has a chemistry similar to the traditional TMA $/ \mathrm{H}_{2} \mathrm{O}$ process. ${ }^{30}$ Here, as an initial attempt to model the liquid-phase chemistry, our simulations will focus on modeling this particular system. Because of the absence of a purging step in this batch liquid-phase process, stoichiometric amounts of precursor are added to each half cycle to achieve monolayer coverage and to prevent unwanted side reactions and uncontrolled growth. Experimentally, there is a large parameter space to explore, including the quantities of precursor injected per cycle, temperature, and the choice of precursors, substrate, and solvent. There are multiple outputs of interest that may vary nonmonotonically with respect to these inputs, including growth rate, roughness, and film density. To completely explore this space through experiments is challenging, thus we wanted to first identify interesting regimes via modeling the liquid-phase chemical layer deposition (CLD) process. This is also, to our knowledge, the first attempt of modeling liquid-phase deposition and as such could have many implications beyond our system of interest.

The aim of this work is to both improve our understanding of the mechanism of the traditional gas-phase alumina ALD process and further extend the model to analyze a liquid-phase layer-by-layer overcoating process relevant to heterogeneous catalysis. In this work, we identify the importance of a dissociation mechanism for TMA deposition on alumina in gasphase ALD and the importance of the precipitation deposition mechanism in the case of liquid-phase overcoating.

\section{COMPUTATIONAL DETAILS}

2.1. Rule-Based Approximation Phase. All major reaction pathways considered in this work for the TMA/ $\mathrm{H}_{2} \mathrm{O}$ process are presented in Figure 1. The deposition rules are informed by mechanistic studies of the TMA $/ \mathrm{H}_{2} \mathrm{O}$ process. ${ }^{1,20,31-34}$ These works show common pathways, despite often studying different surface models with different methods. There is a general agreement about the favorability of the ligand exchange reaction (LER) R1 on hydroxylated surfaces (Figure 1), and R2 was found to directly follow in most studies. Shirazi and Elliott ${ }^{33}$ found that the subsequent LER, R3, was made favorable through a cooperative effect from the neighboring $\mathrm{Me}_{2}$ or $\mathrm{Me}_{1}$ surface species. Other studies did not include any cooperative effect, although there is a disagreement about whether the precursor passes through the planar intermediate $\mathrm{Me}_{1},{ }^{34}$ in contrast to directly forming the tetrahedral product of $\mathrm{R} 4, \mathrm{Me}_{1}^{\prime \prime}{ }^{20} \mathrm{R} 5$ was found to be approximately neutral energetically, with a high activation energy, ranging from 1.0 to $1.6 \mathrm{eV}$, leaving $\mathrm{Me}_{1}$ as the kinetically favored product of LERs. ${ }^{31-33} \mathrm{R} 6$ and R7 are alternatives to R2 and R4, in the case where no hydroxyl groups are nearby. ${ }^{20,32}$ Many studies that consider bare or partially hydroxylated surfaces acknowledge 


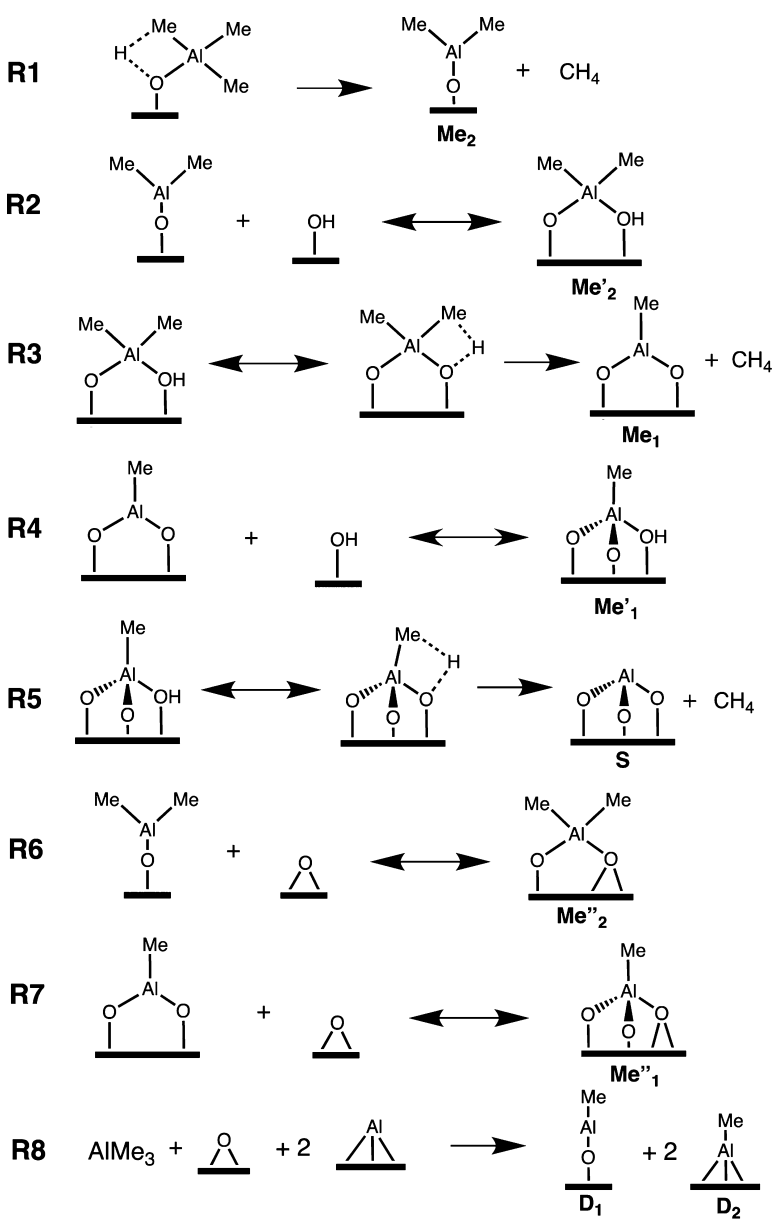

Figure 1. TMA reaction pathways (adapted from Travis and Adomaitis $^{34}$ ).

that TMA can adsorb to oxygen bridges. ${ }^{20,32-34}$ From here, the precursor can irreversibly dissociate into three methyl fragments via R7. Other pathways have been proposed, such as those that model adsorbed $\mathrm{H}_{2} \mathrm{O}$ and TMA coordinated with bridge oxygens, ${ }^{32}$ but these were not explicitly treated in this study.

In contrast to studies that use fixed surfaces, any set of deposition rules, including ours, must accept arbitrary surface atom coordinates and define rules that describe which reactions can happen. However, simple information on particular interatomic distances and angles is not sufficient to generally establish in which range of distances and angles the reactions are favored. In the absence of these additional data, a bridging distance parameter was introduced so that hydroxyl groups within this distance are deemed eligible for a LER $(\mathrm{R} 2+\mathrm{R} 3$ or $\mathrm{R} 4+\mathrm{R} 5$ ). This distance parameter was selected to be $3.5 \AA$, based on a transition-state structure for R2 identified by Weckman and Laasonen ${ }^{31}$ (see calculation in section S1 of the Supporting Information).

The deposition rules attempt to produce a plausible final state, rather than depict a rigorous sequence of events that lead to the final state. Thus, the stable bridged species $\mathrm{Me}_{2}^{\prime}$ is directly formed when a precursor is deposited onto an unhindered hydroxyl with a neighbor within the bridging distance, rather than passing through intermediate states. Because of uncertainty in the relative reaction rates of the dissociation and LERs, the rules alternate attempts at the two types of reaction. Miniature MD relaxations are performed when a surface is apparently saturated, and a surface is deemed saturated if the miniature $\mathrm{MD}$ relaxation does not lead to any further addition. The system is represented by a list of species in the gas phase or in solution and a list of surface atoms ( $\mathrm{Si}, \mathrm{Al}$, $\mathrm{O}, \mathrm{H}$, and ligand) with their coordinates. Precursor ligands are approximated as large single pseudo-atoms. The precursor addition rules and their sequence are shown in Figure 2.

Precursor addition involves evenly sampling from potential structures that maintain the structure's geometry (Figure 1). A precursor addition is deemed successful if there exists a structure with no hard sphere overlap. If multiple successful structures are found, the structure closest to their average is chosen. Dissociation reactions target bare alumina and bridge oxygen sites.

The hydrolysis half cycle is assumed to behave ideally, resulting in the hydrolysis of every ligand present on the

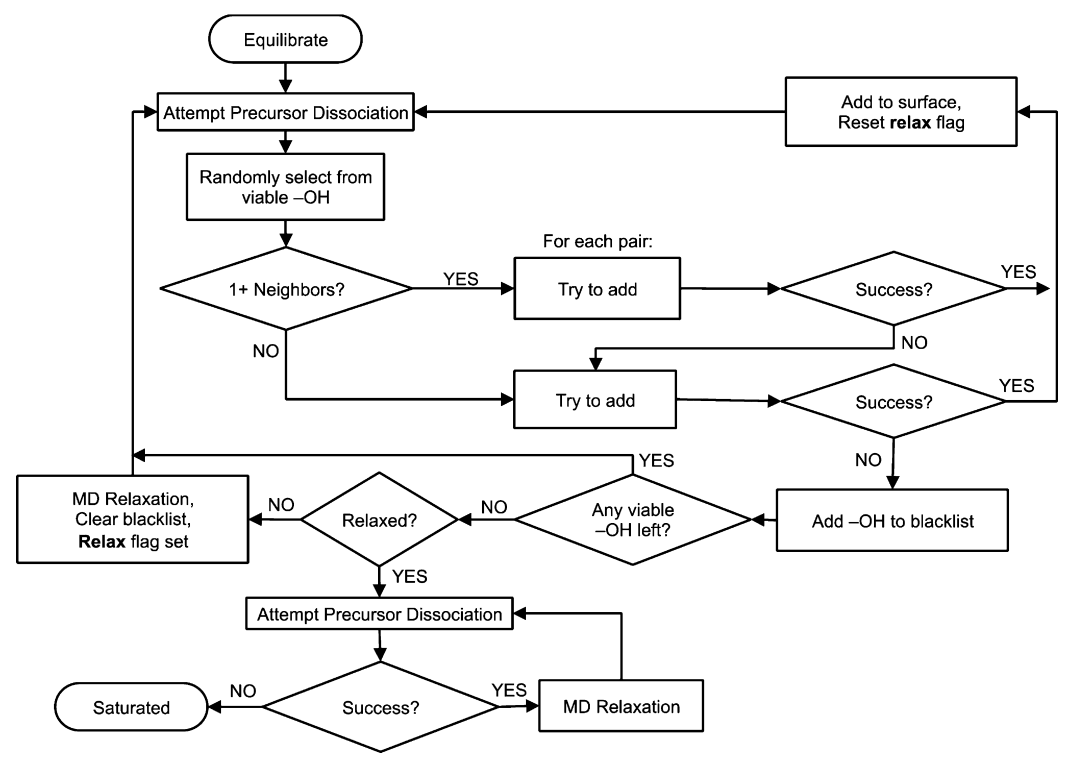

Figure 2. Surface saturation flowchart. $-\mathrm{OH}$ neighbors are hydroxyls within the bridging distance of $3.5 \AA$. 
surface, with no dissociation of water onto bare alumina. Experimentally, this is validated by $\mathrm{C}$ impurities detected at molar concentrations on the order of $1 \% .{ }^{35}$ After the second half cycle, a 200 ps $\mathrm{MD}$ relaxation is performed before beginning the subsequent cycle.

2.2. MD Relaxation Phase. MD relaxations are invoked to produce a realistic equilibrium surface after an approximate surface has been generated from the deposition rules (Figure 2). The MD relaxation phase is governed by a Buckinghamtype pair potential (eq 3), originally developed to treat crystals and melts of alumina and silica. ${ }^{36}$

$$
U_{i j}=\frac{q_{i} q_{j}}{r_{i j}}+D\left(B_{i}+B_{j}\right) \exp \left(\frac{A_{i}+A_{j}-r_{i j}}{B_{i}+B_{j}}\right)+\frac{C_{i} C_{j}}{r_{i j}{ }^{6}}
$$

This potential was expanded to treat hydroxylated surfaces of alumina and silica. ${ }^{37}$ Stiff harmonic bonds were used to keep ligands at a fixed distance while allowing them freedom of motion. An interaction potential for the ligand pseudo-atoms was developed such that they would not overlap with other atoms. This is accomplished with a purely repulsive LennardJones potential with a cutoff at its minimum value. The parameters for these potential functions are found in Tables S1 and S2 in the section S2 of the Supporting Information.

Simulations were carried out using an NVT ensemble in a simulation box with periodic boundaries along $x$ and $y$ directions. $^{38}$ Full relaxations lasted 200 ps with a 0.5 fs timestep and sampling taken over a 100 ps period. A twodimensional correction was applied to the Ewald summation of Coulombic forces. A Nosé-Hoover thermostat was implemented, maintaining a temperature of $450 \mathrm{~K}$. The cutoff for Buckingham interactions was $8 \AA$, and the cutoff for Coulombic interactions was $13 \AA$. An artificial force beginning $5 \AA$ above the surface repelled any molecules that might break free from the surface and attempt to escape the simulation box. This force was modeled as a harmonic spring acting purely in the vertical dimension. Simulations were performed using the MD package, LAMMPS, and results were visualized using OVITO. ${ }^{38,39}$

The initial alumina surface model was prepared by annealing an alumina model after replacing random $\mathrm{Al}$ atoms with $\mathrm{H}$ atoms, as described in $\mathrm{Hu}$ et al. ${ }^{29}$ The surface had 2265 atoms, $6 \% \mathrm{H}$, a surface $-\mathrm{OH}$ density of $2.1 \mathrm{OH} \mathrm{nm}{ }^{-2}$, and the simulation box had dimensions $a=b=36.8964 \AA$ and $c=70.0$ $\AA$, including a vacuum space of $50 \AA$ above the surface. A hydroxylated silica model prepared by Sandupatla et al. ${ }^{40}$ with 5 $\mathrm{OH} \mathrm{nm}{ }^{-2}$ was relaxed for 300 ps under the Matsui potential before being used in MD simulations.

2.3. Liquid-Phase Implementation. The consequences of possibly adding insufficient or excess precursors due to the batch configuration of the liquid-phase experiments required additions to the gas-phase model. In particular, a list had to be used to keep track of the species in solution. The equilibration routine had to analyze the list of species in the solution and the surface and terminated only when no reactions were possible. The species and reactions that were considered are presented in Tables 1 and 2 .

The gas-phase ALD model contains four classes of species: $* \mathrm{~L}$, $* \mathrm{OH}, \mathrm{H}_{2} \mathrm{O}$, and $\mathrm{AlL}_{3}$ (where $\mathrm{L}=-\mathrm{CH}_{3}$ in the gas-phase model and $-\mathrm{O}$-sec-Bu in the liquid-phase model). In the liquidphase CLD case, the possibility of excess precursor reacting with $\mathrm{H}_{2} \mathrm{O}$ in solution leads to a partially hydrolyzed precursor, which could further react to form oligomers. Thus, the partially hydrolyzed precursor and the oligomerized precursor were new
Table 1. Modeled Species and Their Possible Reactions ${ }^{a}$

\begin{tabular}{llc}
\multicolumn{1}{c}{ species } & \multicolumn{1}{c}{ name } & reactions \\
$\mathrm{AlL}_{3}$ & precursor & $1,3,4$ \\
$\mathrm{H}_{2} \mathrm{O}$ & water & 2,3 \\
$* \mathrm{OH}$ & surface hydroxyl & 1,2 \\
$* \mathrm{~L}$ & surface ligand & 1,2 \\
$\mathrm{AlL}_{x}(\mathrm{OH})_{y}$ & partially hydrolyzed precursor & $1,3,4$ \\
$\mathrm{Al}_{x} \mathrm{O}_{y} \mathrm{~L}_{z}(\mathrm{OH})_{n}$ & precipitate (oligomerized precursor) & 3,4 \\
${ }^{a} \mathrm{Reaction} \mathrm{numbers}$ & \\
\hline
\end{tabular}

Table 2. Modeled Reactions ${ }^{a}$

\begin{tabular}{|c|c|c|}
\hline \# & stoichiometry & name \\
\hline 1 & $\mathrm{AlL}_{x}(\mathrm{OH})_{y}+* \mathrm{OH} \rightarrow * \mathrm{AlL}_{x-1}(\mathrm{OH})_{y}$ & $\begin{array}{l}\text { surface ligand exchange (R1, } \\
\text { R2) }\end{array}$ \\
\hline 2 & $\mathrm{AlL}_{x}(\mathrm{OH})_{y}+2 * \mathrm{OH} \rightarrow * \mathrm{AlL}_{x-2}(\mathrm{OH})_{y}$ & $\begin{array}{l}\text { surface ligand exchange (R3, } \\
\text { R4) }\end{array}$ \\
\hline 3 & $\mathrm{AlL}_{3}+3 * \mathrm{OH} \rightarrow * \mathrm{Al}$ & surface ligand exchange (R5) \\
\hline 4 & $\mathrm{AlL}_{x}(\mathrm{OH})_{y}+* \mathrm{OH} \rightarrow * \mathrm{AlL}_{x}(\mathrm{OH})_{y}$ & surface dissociation (R8) \\
\hline 5 & $\mathrm{H}_{2} \mathrm{O}+* \mathrm{~L} \rightarrow * \mathrm{OH}$ & surface hydrolysis \\
\hline 6 & $\begin{array}{l}\mathrm{Al}_{x} \mathrm{O}_{y} \mathrm{~L}_{z}(\mathrm{OH})_{n}+\mathrm{H}_{2} \mathrm{O} \rightarrow \\
\mathrm{Al}_{x} \mathrm{O}_{y} \mathrm{~L}_{z-1}(\mathrm{OH})_{n+1}\end{array}$ & $\begin{array}{l}\text { precursor hydrolysis/ } \\
\text { precipitate hydrolysis }\end{array}$ \\
\hline t & $\begin{array}{c}\mathrm{Al}_{a} \mathrm{O}_{b} \mathrm{~L}_{c}(\mathrm{OH})_{d}+\mathrm{Al}_{x} \mathrm{O}_{y} \mathrm{~L}_{z}(\mathrm{OH})_{n} \rightarrow \\
\mathrm{Al}_{a+x} \mathrm{O}_{b+y+1} \mathrm{~L}_{c+z-1}(\mathrm{OH})_{d+n-1}\end{array}$ & $\begin{array}{l}\text { precursor agglomeration } \\
\text { precipitate agglomeration }\end{array}$ \\
\hline
\end{tabular}

${ }^{a_{T}}$ The $\mathrm{L}-\mathrm{H}$ byproduct for each reaction is omitted from the stoichiometry column, as it is an inert species which is not modeled. $\mathrm{R} 1-\mathrm{R} 8$ refer to the pathways described in Figure 1. The symbol * designates a surface bond.

species for the liquid-phase model, with the former allowed to participate in surface ligand exchange. Although the oligomers could form separate particles, it was assumed that they could precipitate onto the surface of the growing film. This was modeled by a precipitation parameter. This final assumption was supported by experiments, where no free alumina was observed at the end of the synthesis. ${ }^{30}$

The possibility of multiple reactions being possible at once raises important questions of kinetics and mass transfer. In the absence of this information, we treated all reactions as equally likely, with the equilibration process consisting of random selection of pairs of reactive species, reacting when the rules permitted it, until no more reactions were possible.

As mentioned, the precipitate could deposit onto the surface. This was done by constructing a plausible molecule, giving an atomic composition, and orienting it in a random direction at a random point on the surface. In reality, the rate of this process is a function of many unknown concentrations, reaction rates, and mass-transfer coefficients. We approximated this process using a single unknown factor, expressed as a probability of precipitation per cycle (referred to above as the precipitation parameter).

The last addition to the liquid-phase model was a representation of solvent molecules during the $\mathrm{MD}$ relaxation. After a half reaction had reached equilibrium, the MD relaxation phase was preceded by filling the simulation box with randomly oriented solvent molecules (sec-butanol) up to the experimental density (Figure 3). A repulsive-only harmonic spring wall was placed $15 \AA$ above the surface to bound the motion of solvent molecules. Force fields for both the solvent interactions and the interface interactions were taken from Jorgensen et al. ${ }^{41}$ and Johnston et al., ${ }^{42}$ respectively. An example of different phases of CLD simulation is presented in Figure 3. 

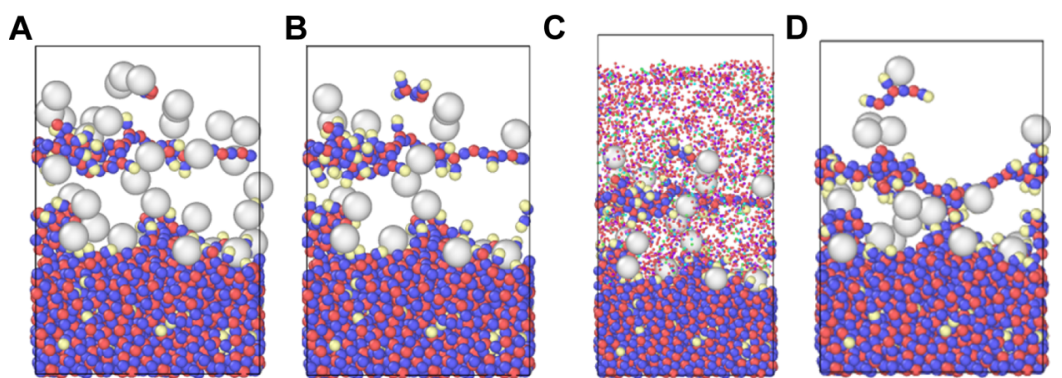

Figure 3. CLD simulation box snapshots. (A) Precursor injection, (B) water injection, (C) solvent addition and surface relaxation, and (D) surface after relaxation. Color code: red $=$ aluminum, blue $=$ oxygen, yellow $=$ hydrogen, and white $=\mathrm{O}$-sec-Bu ligand. Solvent atoms in panel $\mathrm{C}$ are shown with a reduced size.
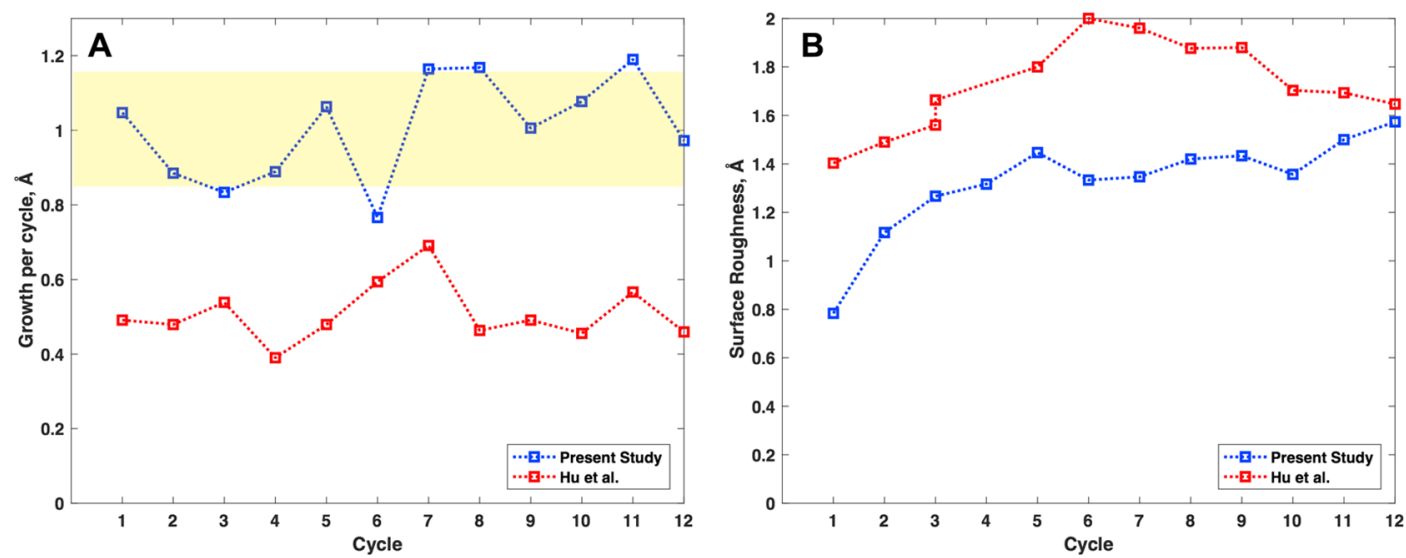

Figure 4. ALD simulations. (A) Height of film growth per cycle and (B) roughness per cycle. Present results compared to Hu et al. ${ }^{29}$ The range of growth per cycle commonly observed experimentally is highlighted in yellow. ${ }^{43}$
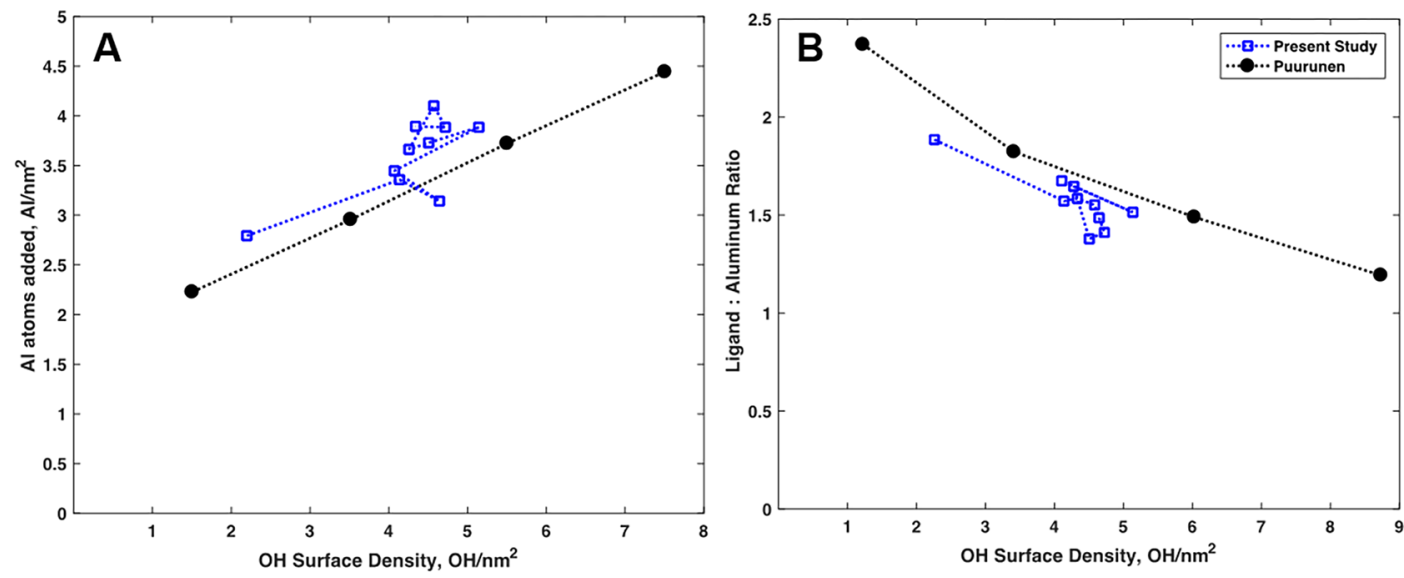

Figure 5. Growth properties as a function of surface hydroxyl density. concentration. Present results compared to data from Puurunen. ${ }^{45,46}$

\section{RESULTS AND DISCUSSION}

3.1. Atomistic Simulation of the $\mathrm{TMA} / \mathrm{H}_{2} \mathrm{O}$ ALD

Process. The first case study we modeled was the growth of TMA $/ \mathrm{H}_{2} \mathrm{O}$ over a hydroxylated alumina surface at room temperature. Predicted growth and roughness per cycle are plotted in Figure 4 as a function of the ALD cycle. The results are compared to those of $\mathrm{Hu}$ et al., which studied the same physical system, ${ }^{29}$ and to the experimentally observed growth rate, that is, between 0.9 and $1.1 \AA /$ cycle $^{43}$ (yellow zone) (Figure 4A). The increased growth per cycle that we predict relative to $\mathrm{Hu}$ et al. ${ }^{29}$ was largely a consequence of introducing the dissociation reaction and more closely matched exper- (a) $\mathrm{Al}$ atoms added vs $\mathrm{OH}$ density. (b) Ligand/Al ratio vs surface $\mathrm{OH}$ imental values, suggesting that the dissociation reaction may be an important inclusion to correctly predict experimental results. Our trend for surface roughness is similar to that of $\mathrm{Hu}$ et al. ${ }^{29}$ as it increased with the number of cycles, which was suggestive of the island-growth mode (Figure 4B).

Similarly to $\mathrm{Hu}$ et al., ${ }^{29}$ our initial surface has a lower surface $\mathrm{OH}$ density than typical ALD conditions. ${ }^{44}$ However, over multiple cycles, this value increases until it reached a stable value of $4.5 \mathrm{OH} \mathrm{nm}{ }^{-2}$. By monitoring how the deposition changes over multiple cycles, it was possible to reproduce several experimental trends, including the number of aluminum atoms added (Figure 5A) or the ratio of aluminum atoms 
added to ligands added for a given surface $\mathrm{OH}$ density (Figure $5 B)$.

Figure 6 shows predicted $\mathrm{Al}_{2} \mathrm{O}_{3}$ coating growth on alumina and silica represented as the number of $\mathrm{Al}$ atoms added to the

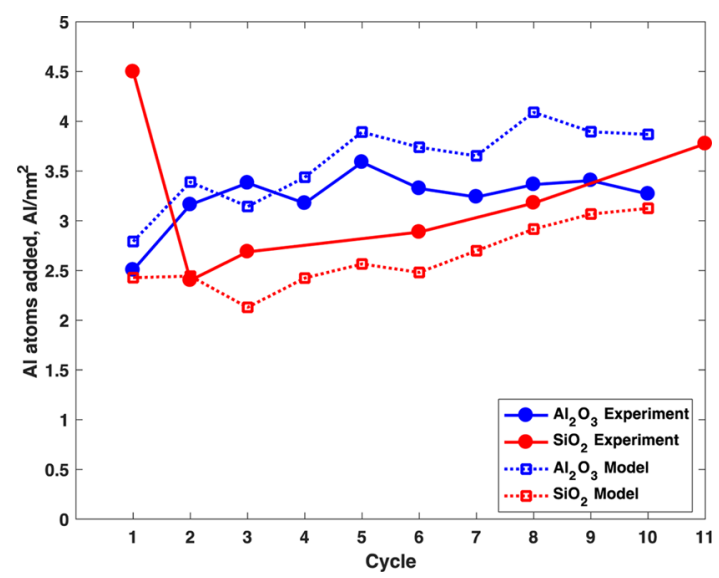

Figure 6. Predicted and measured alumina ALD coating growths on an alumina (blue) and silica (red) surface. Predictions are depicted by squares and dotted lines. Measurements of $\mathrm{Al}$ added on silica ${ }^{47}$ are depicted by red circles and solid lines. Experimental values for $\mathrm{Al}$ added over alumina (blue circles and solid lines) inferred from Puurunen's empirical model, ${ }^{45,46}$ which related the number of $\mathrm{Al}$ atoms added to surface $\mathrm{OH}$ coverage.

substrate. The simulations over silica are compared to the experimental silica ALD results of Delabie et al. ${ }^{47}$ The predicted growth over alumina is compared to the expected cycle-by-cycle growth data of alumina that was obtained by using the surface $\mathrm{OH}$ density with the empirical relation given by Puurunen ${ }^{45,46}$ (see Figure 5A). A characteristic difference in coating a silica substrate compared to alumina was the initial decrease in deposited aluminum, followed by a gradual increase. This trend is explained by Delabie et al. ${ }^{47}$ as a consequence of the inability for $\mathrm{Si}-\mathrm{CH}_{3}$ bonds to be hydrolyzed, creating an inert surface area after the first half cycle. As the ratio of aluminum to silicon atoms on the surface gradually increases after further ALD cycles, the inert fraction of the surface decreases and the growth rate increases. However, our inclusion of a silica surface model that forbade the hydrolysis of $\mathrm{Si}-\mathrm{CH}_{3}$ bonds led to a very limited decrease over the first two cycles that was not as pronounced as the experimental trend measured by Delabie et al., ${ }^{47}$ suggesting that other effects are perhaps at play.

Ligand size was found to have a large impact on the growth rate. Increasing the ligand radius from 2.0 to $2.4 \AA$, consistent with using a triethylaluminum precursor, led to a decrease in the growth rate from approximately 1.0 to $0.6 \AA /$ cycle, with no significant difference in the surface roughness.

Sensitivity to the bridging distance parameter was also investigated; however, no significant deviations in any model outputs were observed between tests at $2.5,3.5$, and $4.5 \AA$. This speaks to the relative dominance of the dissociation reaction, which is not impacted by the bridging parameter, at the low surface $\mathrm{OH}$ concentrations tested in this study. This is in agreement with previous reports as the dominance of dissociation at low and moderate surface $\mathrm{OH}$ concentrations has been predicted by Travis and Adomaitis ${ }^{34}$ and experimentally suggested by Guerra-Nuñez et al. ${ }^{48}$

3.2. Atomistic Simulation of the Liquid-Phase CLD. As mentioned above, the case study developed for liquid-phase simulation was the $\mathrm{Al}(\mathrm{O}-\mathrm{sec}-\mathrm{Bu})_{3} / \mathrm{H}_{2} \mathrm{O}$ process solvated in secbutanol, recently developed by Héroguel et al. ${ }^{30}$

A significant portion of the CLD was found to come from the precipitation mechanism, rather than the traditional ALD pathway. This is evidenced by a lack of sensitivity to the ligand size, which the gas-phase model is very sensitive to. This growth mode leads to rough surface films with high porosity (Figure 3), relative to the analogous ALD case (see comparisons in Figure 7).

The model revealed that growth rates are sensitive to the input stoichiometry (Figure 8). In particular, the growth and roughness can be multiplied by 10 by decreasing the amount of water injected per cycle. This is understood as using a "nonideal" ratio of precursors to encourage the precipitate deposition growth mode, evidenced by the significant increase in roughness. The CLD overcoat obtained experimentally ${ }^{30}$ shows much greater porosity than ALD overcoats, which is also qualitatively captured by the model. Given the very limited number of studies using simulations to predict liquid-phase sol-gel chemistry, these results that predict similar trends to those observed experimentally demonstrate that such efforts could serve as a tool to guide experimentalists during thin-film synthesis in solution.

\section{CONCLUSIONS}

We have successfully expanded a simulation method for modeling ALD involving the combination of an empirical, rule-based deposition approach with a MD relaxation step. This method is a simpler alternative to the LKMC strategy, which requires an intensive DFT investigation to fully characterize all types of possible changes in atomic coordinates. By incorporating several recently reported mechanistic features for the TMA $/ \mathrm{H}_{2} \mathrm{O}$ process into our ALD modeling algorithm, we improved the ability of the model to predict several
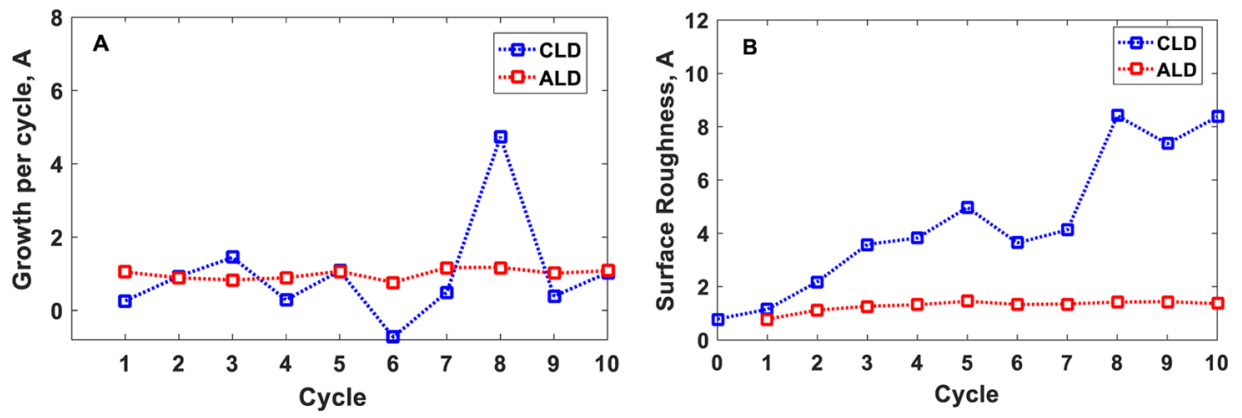

Figure 7. CLD simulations. (A) Cumulative height growth and (B) roughness per cycle. 

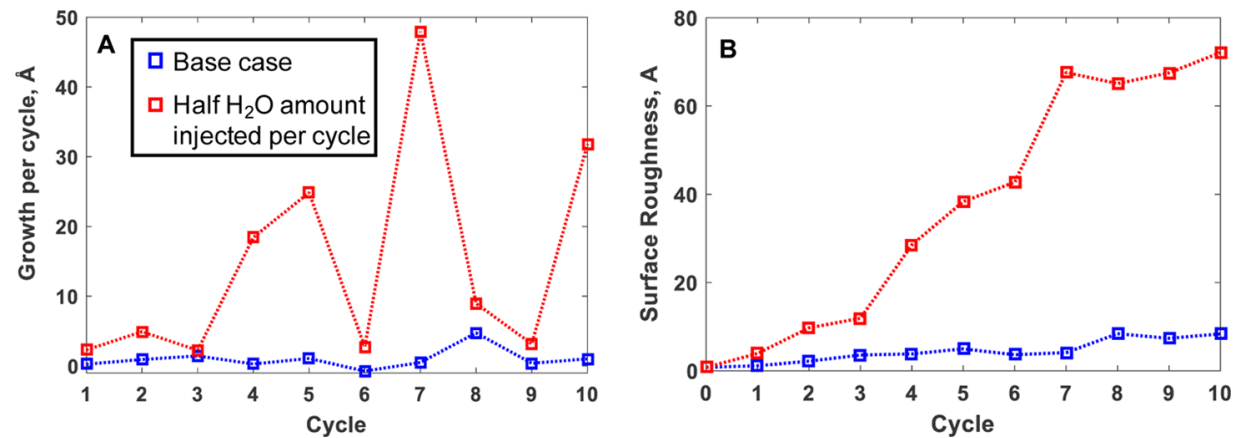

Figure 8. Effect of different injection stoichiometries. (A) Cumulative height growth per cycle and (B) roughness per cycle. Blue: base case. Red: half amount of water injected per cycle.

variables. Importantly, a stable growth rate on alumina of approximately $1.0 \AA$ /cycle at $450 \mathrm{~K}$ was predicted, successfully reproducing experimental results. Furthermore, we expanded our approach to modeling liquid-phase thin-film deposition, which is of growing importance in the sol-gel chemistry research. Our ability to qualitatively predict trends between input variables (e.g., stoichiometry) and film growth or roughness suggests that this model can help guide future experimental design.

\section{ASSOCIATED CONTENT}

\section{S Supporting Information}

The Supporting Information is available free of charge on the ACS Publications website at DOI: 10.1021/acs.jpcc.8b00197.

Calculation of the bridging distance parameter between two hydroxyl groups and description of the potential functions parameters (PDF)

\section{AUTHOR INFORMATION}

\section{Corresponding Author}

*E-mail: jeremy.luterbacher@epfl.ch.

ORCID $\odot$

Chiara Saggese: 0000-0002-5481-0442

Jeremy S. Luterbacher: 0000-0002-0967-0583

\section{Present Addresses}

${ }^{\dagger}$ Chemical Engineering Department, Stanford University, Stanford, California-94305, USA.

${ }^{\ddagger}$ Mechanical Engineering Department, Stanford University, Stanford, California-94305, USA.

\section{Notes}

The authors declare no competing financial interest.

\section{ACKNOWLEDGMENTS}

We acknowledge funding from the Swiss National Science Foundation through grant PYAPP2_154281 and from EPFL. K.S.B. acknowledges the support from Dartmouth College's James B. Reynolds Scholarship. Dr. C.S. acknowledges the support from Swiss Government Excellence Scholarship.

\section{REFERENCES}

(1) Puurunen, R. L. Surface Chemistry of Atomic Layer Deposition: A Case Study for the Trimethylaluminum/Water Process. J. Appl. Phys. 2005, 97, 121301.

(2) Pinna, N.; Knez, M. Atomic Layer Deposition of Nanostructured Materials; John Wiley \& Sons, 2012.

(3) George, S. M. Atomic Layer Deposition: An Overview. Chem. Rev. 2010, 110, 111-131.
(4) Sherman, A. Atomic Layer Deposition for Nanotechnology: An Enabling Process for Nanotechnology Fabrication; Ivoryton Press, 2008.

(5) Kääriäinen, T.; Cameron, D.; Kääriäinen, M.-L.; Sherman, A. Atomic Layer Deposition: Principles, Characteristics, and Nanotechnology Applications; John Wiley \& Sons, 2013.

(6) Hwang, C. S. Atomic Layer Deposition for Semiconductors; Springer, 2014.

(7) Lu, J.; Fu, B.; Kung, M. C.; Xiao, G.; Elam, J. W.; Kung, H. H.; Stair, P. C. Coking-and Sintering-Resistant Palladium Catalysts Achieved through Atomic Layer Deposition. Science 2012, 335, 1205-1208.

(8) O’Neill, B. J.; Jackson, D. H. K.; Crisci, A. J.; Farberow, C. A.; Shi, F.; Alba-Rubio, A. C.; Lu, J.; Dietrich, P. J.; Gu, X.; Marshall, C. L.; Stair, P. C.; Elam, J. W.; Miller, J. T.; Ribeiro, F. H.; Voyles, P. M.; Greeley, J.; Mavrikakis, M.; Scott, S. L.; Kuech, T. F.; Dumesic, J. A. Stabilization of Copper Catalysts for Liquid-Phase Reactions by Atomic Layer Deposition. Angew. Chem. 2013, 125, 14053-14057.

(9) Lee, I.; Zhang, Q.; Ge, J.; Yin, Y.; Zaera, F. Encapsulation of Supported Pt Nanoparticles with Mesoporous Silica for Increased Catalyst Stability. Nano Res. 2011, 4, 115-123.

(10) Bo, Z.; Eaton, T. R.; Gallagher, J. R.; Canlas, C. P.; Miller, J. T.; Notestein, J. M. Size-Selective Synthesis and Stabilization of Small Silver Nanoparticles on $\mathrm{TiO} 2$ Partially Masked by SiO2. Chem. Mater. 2015, 27, 1269-1277.

(11) Johnson, R. W.; Hultqvist, A.; Bent, S. F. A Brief Review of Atomic Layer Deposition: From Fundamentals to Applications. Mater. Today 2014, 17, 236-246.

(12) Ritala, M.; Niinistö, J. Industrial Applications of Atomic Layer Deposition. ECS Trans. 2009, 25, 641-652.

(13) Zhang, F.; Carrier, X.; Krafft, J.-M.; Yoshimura, Y.; Blanchard, J. Insight into the Structure and Localization of the Titania Overlayer in TiO2-Coated SBA-15 Materials. New J. Chem. 2010, 34, 508-516.

(14) Santacesaria, E.; Cozzolino, M.; Di Serio, M.; Venezia, A. M.; Tesser, R. Vanadium Based Catalysts Prepared by Grafting: Preparation, Properties and Performances in the ODH of Butane. Appl. Catal., A 2004, 270, 177-192.

(15) Yan, W.; Mahurin, S. M.; Overbury, S. H.; Dai, S. Nanoengineering Catalyst Supports Via Layer-by-Layer Surface Functionalization. Top. Catal. 2006, 39, 199-212.

(16) Baca, M.; de la Rochefoucauld, E.; Ambroise, E.; Krafft, J.-M.; Hajjar, R.; Man, P. P.; Carrier, X.; Blanchard, J. Characterization of Mesoporous Alumina Prepared by Surface Alumination of SBA-15. Microporous Mesoporous Mater. 2008, 110, 232-241.

(17) Iengo, P.; Di Serio, M.; Sorrentino, A.; Solinas, V.; Santacesaria, E. Preparation and Properties of New Acid Catalysts Obtained by Grafting Alkoxides and Derivatives on the Most Common Supports Note I-Grafting Aluminium and Zirconium Alkoxides and Related Sulphates on Silica. Appl. Catal., A 1998, 167, 85-101.

(18) Widjaja, Y.; Musgrave, C. B. Quantum Chemical Study of the Mechanism of Aluminum Oxide Atomic Layer Deposition. Appl. Phys. Lett. 2002, 80, 3304-3306. 
(19) Dillon, A. C.; Ott, A. W.; Way, J. D.; George, S. M. Surface Chemistry of $\mathrm{Al} 2 \mathrm{O} 3$ Deposition Using $\mathrm{Al}(\mathrm{CH} 3) 3$ and $\mathrm{H} 2 \mathrm{O}$ in a Binary Reaction Sequence. Surf. Sci. 1995, 322, 230-242.

(20) Elliott, S. D.; Greer, J. C. Simulating the Atomic Layer Deposition of Alumina from First Principles. J. Mater. Chem. 2004, 14, 3246-3250.

(21) Pan, D.; Ma, L.; Xie, Y.; Jen, T. C.; Yuan, C. On the Physical and Chemical Details of Alumina Atomic Layer Deposition: A Combined Experimental and Numerical Approach. J. Vac. Sci. Technol., A 2015, 33, 021511.

(22) King, P. J.; Werner, M.; Chalker, P. R.; Jones, A. C.; Aspinall, H. C.; Basca, J.; Wrench, J. S.; Black, K.; Davies, H. O.; Heys, P. N. Effect of Deposition Temperature on the Properties of $\mathrm{CeO} 2$ Films Grown by Atomic Layer Deposition. Thin Solid Films 2011, 519, 4192-4195.

(23) Rai, V. R.; Vandalon, V.; Agarwal, S. Influence of Surface Temperature on the Mechanism of Atomic Layer Deposition of Aluminum Oxide Using an Oxygen Plasma and Ozone. Langmuir 2011, 28, 350-357.

(24) Wind, R. A.; George, S. M. Quartz Crystal Microbalance Studies of Al2O3 Atomic Layer Deposition Using Trimethylaluminum and Water at $125{ }^{\circ} \mathrm{C}$. J. Phys. Chem. A 2010, 114, 1281-1289.

(25) Krajewski, T.; Guziewicz, E.; Godlewski, M.; Wachnicki, L.; Kowalik, I. A.; Wojcik-Glodowska, A.; Lukasiewicz, M.; Kopalko, K.; Osinniy, V.; Guziewicz, M. The Influence of Growth Temperature and Precursors' Doses on Electrical Parameters of Zno Thin Films Grown by Atomic Layer Deposition Technique. Microelectron. J. 2009, 40, 293-295.

(26) Elliott, S. D.; Dey, G.; Maimaiti, Y.; Ablat, H.; Filatova, E. A.; Fomengia, G. N. Modeling Mechanism and Growth Reactions for New Nanofabrication Processes by Atomic Layer Deposition. Adv. Mater. 2015, 28, 5367-5380.

(27) Afshar, A.; Cadien, K. C. Growth Mechanism of Atomic Layer Deposition of Zinc Oxide: A Density Functional Theory Approach. Appl. Phys. Lett. 2013, 103, 251906.

(28) Shirazi, M.; Elliott, S. D. Atomistic Kinetic Monte Carlo Study of Atomic Layer Deposition Derived from Density Functional Theory. J. Comput. Chem. 2014, 35, 244-259.

(29) Hu, Z.; Shi, J.; Turner, C. H. Molecular Dynamics Simulation of the Al2O3 Film Structure During Atomic Layer Deposition. Mol. Simul. 2009, 35, 270-279.

(30) Héroguel, F.; Le Monnier, B. P.; Brown, K. S.; Siu, J. C.; Luterbacher, J. S. Catalyst Stabilization by Stoichiometrically Limited Layer-by-Layer Overcoating in Liquid Media. Appl. Catal., B 2017, $218,643-649$.

(31) Weckman, T.; Laasonen, K. First Principles Study of the Atomic Layer Deposition of Alumina by TMA-H2O-Process. Phys. Chem. Chem. Phys. 2015, 17, 17322-17334.

(32) Sandupatla, A. S.; Alexopoulos, K.; Reyniers, M.-F.; Marin, G. B. $\mathrm{Ab}$ Initio Investigation of Surface Chemistry of Alumina ALD on Hydroxylated $\Gamma$-Alumina Surface. J. Phys. Chem. C 2015, 119, 1305013061.

(33) Shirazi, M.; Elliott, S. D. Cooperation between Adsorbates Accounts for the Activation of Atomic Layer Deposition Reactions. Nanoscale 2015, 7, 6311-6318.

(34) Travis, C. D.; Adomaitis, R. A. Modeling Alumina Atomic Layer Deposition Reaction Kinetics During the Trimethylaluminum Exposure. Theor. Chem. Acc. 2014, 133, 1414.

(35) Rahtu, A.; Alaranta, T.; Ritala, M. In Situ Quartz Crystal Microbalance and Quadrupole Mass Spectrometry Studies of Atomic Layer Deposition of Aluminum Oxide from Trimethylaluminum and Water. Langmuir 2001, 17, 6506-6509.

(36) Matsui, M. A Transferable Interatomic Potential Model for Crystals and Melts in the System CaO-MgO-Al2O3-SiO2. Mineral. Mag. 1994, 58A, 571-572.

(37) Adiga, S. P.; Zapol, P.; Curtiss, L. A. Structure and Morphology of Hydroxylated Amorphous Alumina Surfaces. J. Phys. Chem. C 2007, 111, 7422-7429.

(38) Plimpton, S. Fast Parallel Algorithms for Short-Range Molecular Dynamics. J. Comput. Phys. 1995, 117, 1-19.
(39) Stukowski, A. Visualization and Analysis of Atomistic Simulation Data with Ovito-the Open Visualization Tool. Modell. Simul. Mater. Sci. Eng. 2009, 18, 015012.

(40) Sandupatla, A. S.; Alexopoulos, K.; Reyniers, M.-F.; Marin, G. B. Dft Investigation into Alumina Ald Growth Inhibition on Hydroxylated Amorphous Silica Surface. J. Phys. Chem. C 2015, 119, 1838018388.

(41) Jorgensen, W. L.; Maxwell, D. S.; Tirado-Rives, J. Development and Testing of the Opls All-Atom Force Field on Conformational Energetics and Properties of Organic Liquids. J. Am. Chem. Soc. 1996, $118,11225-11236$

(42) Johnston, K.; Herbers, C. R.; van der Vegt, N. F. A. Development of Classical Molecule-Surface Interaction Potentials Based on Density Functional Theory Calculations: Investigation of Force Field Representability. J. Phys. Chem. C 2012, 116, 1978119788.

(43) Puurunen, R. L. Growth Per Cycle in Atomic Layer Deposition: Real Application Examples of a Theoretical Model. Chem. Vap. Deposition 2003, 9, 327-332.

(44) Zhuravlev, L. T. The Surface Chemistry of Amorphous Silica. Zhuravlev Model. Colloids Surf., A 2000, 173, 1-38.

(45) Puurunen, R. L.; Lindblad, M.; Root, A.; Krause, A. O. I. Successive Reactions of Gaseous Trimethylaluminium and Ammonia on Porous Alumina. Phys. Chem. Chem. Phys. 2001, 3, 1093-1102.

(46) Puurunen, R. L. Correlation between the Growth-Per-Cycle and the Surface Hydroxyl Group Concentration in the Atomic Layer Deposition of Aluminum Oxide from Trimethylaluminum and Water. Appl. Surf. Sci. 2005, 245, 6-10.

(47) Delabie, A.; Sioncke, S.; Rip, J.; Van Elshocht, S.; Pourtois, G.; Mueller, M.; Beckhoff, B.; Pierloot, K. Reaction Mechanisms for Atomic Layer Deposition of Aluminum Oxide on Semiconductor Substrates. J. Vac. Sci. Technol., A 2012, 30, $01 \mathrm{~A} 127$.

(48) Guerra-Nuñez, C.; Döebeli, M.; Michler, J.; Utke, I. Reaction and Growth Mechanisms in Al2O3 Deposited Via Atomic Layer Deposition: Elucidating the Hydrogen Source. Chem. Mater. 2017, 29, 8690-8703. 